\title{
Benefits of up-wave measurements in linear short-term wave forecasting for wave energy applications
}

\author{
Francesco Paparella ${ }^{1}$, Kieran Monk ${ }^{2,3}$, Victor Winands ${ }^{4}$ \\ M.F.P Lopes ${ }^{3}$, Daniel Conley ${ }^{2}$ and John V. Ringwood ${ }^{1}$
}

\begin{abstract}
The real-time control of wave energy converters requires the prediction of the wave elevation at the location of the device in order to maximize the power extracted from the waves. One possibility is to predict the future wave elevation by combining its past history with the spatial information coming from a sensor which measures the free surface elevation upwave of the wave energy converter. As an application example, the paper focuses on the prediction of the wave elevation inside the chamber of the oscillating water column (OWC) for the PICO OWC plant in the Azores, and two different sensors for the measurement of the free surface elevation up-wave of the oscillating water column were tested. The study showed that the use of the additional information coming from the up-wave sensor does not significantly improve the linear prediction of the chamber wave elevation given by a forecasting model based only on the past values of the chamber wave elevation.
\end{abstract}

\section{INTRODUCTION}

Wave energy converters (WECs) are usually designed to efficiently extract energy from the sea for a limited range of frequencies, usually located around the resonance of the device. In order to realize an efficient energy extraction for a broader range of frequencies, a control system can be designed [1], [2]. Real-time optimal control can be derived from a controller designed in the frequency domain, but it involves noncausal transfer functions which can be implemented only if the future motion of the device or the incident wave elevation are known [1]- [2]. Usually, prediction of the wave elevation is based on a spatial reconstruction of the wave field starting from a set of sensors located in the proximity of the WEC, as shown in Figure 1b [3]. Another possible solution is to predict the wave elevation based only on its past history, as shown in Figure 1a. Regarding the spatial approach for wave prediction, in [4] digital filters were deployed for the real time prediction of waves incident upon a wave energy device. In [5] the remote wave profile is measured by means of a nautical radar, and is propagated in time and space in order to predict the wave field and estimate the motion of a vessel. In [6], a shallow angle LIDAR was

\footnotetext{
${ }^{1}$ Francesco Paparella and John Ringwood are with the Center for Ocean Energy Research (COER), National University of Ireland Maynooth, Co. Kildare, Ireland (e-mail: fpaparella@eeng.nuim.ie, john.ringwood@eeng.nuim.ie)

${ }^{2}$ Kieran Monk and Daniel Conley are with the School of Marine Science and Engineering, University of Plymouth, Plymouth, PL48AA, UK (email: Kieran.Monk@plymouth.ac.uk, daniel.conley@plymouth.ac.uk)

${ }^{3}$ Kieran Monk and M.F.P Lopes are with the WAVEC Offshore Renewables, Lisbon, 1400-119, Portugal (email: kieran@wavec.org, mlopes@wavec.org)

${ }^{4}$ Victor Winands is with Duespan Wave Engineering (email: Duespan@googlemail.com)
}

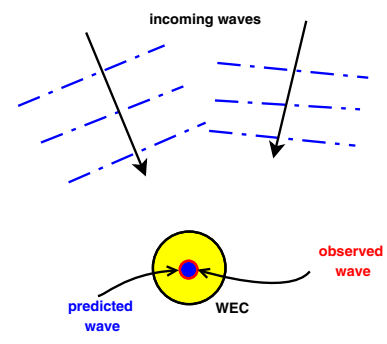

(a)

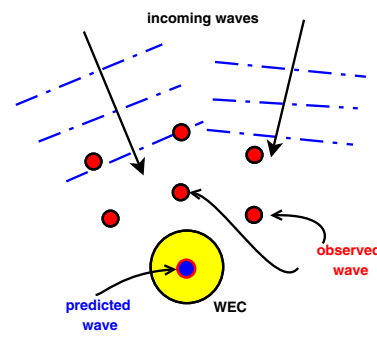

(b)
Fig. 1. Wave prediction based on one-single point of measurements (a) and wave prediction based on spatial reconstruction from array of sensors (b)

adopted in order to measure the time evolution of spatial profiles of sea waves over an extended region of several hundred meters. In [7] the hydrodynamics of the chamber of an oscillating water column (OWC) is predicted by means of a neural network based on the measurements of the incident wave elevation.

Regarding the time series approach for wave prediction, in [3] autoregressive (AR) models are proposed and validated against real observations. In [8] adaptive filters based on autoregressive models for wave prediction are deployed. In [9] a robust control for wave energy devices based on the prediction of the wave elevation is implemented. For the wave prediction, a hybrid Kautz/AR predictive model as well as a purely predictive Kautz model are proposed. In [10] neural networks for the estimation of the wave excitation force were trained and results compared to other methods.

This paper proposes an AutoRegressive eXogenous input (ARX) model, which extends the AR model with the inclusion of the additional information coming from the measurement of the free surface elevation up-wave of the WEC. The up-wave elevation is considered to be an exogenous input of the model. The ARX models proposed are validated against real observations coming from the Pico WEC plant located on the Portuguese island of Pico in the Azores archipelago [7]. In particular, two type of sensors for the up-wave elevation have been tested. The real data available are discussed in section 2, while the forecasting models proposed are outlined in section 3. Finally the results are discussed in section 4 .

\section{Available Data}

This paper proposes a strategy for forecasting the wave elevation inside the chamber of the PICO OWC by a 
combination of its past values and the measurement of the free surface elevation up-wave of the WEC. A sonar sensor for recording the chamber wave elevation is mounted on the roof of the chamber, while two different sensors for the measurement of the free surface elevation around 60 $\mathrm{m}$ in front of the Pico OWC chamber wall are deployed [7]. During the first deployment period, the "Aquadopp" hydrostatic pressure sensor and ADCM (Acoustic Doppler Current Meter) unit was utilized to measure the hydrodynamics of the free surface elevation up-wave of the WEC. Also, an alternative sensor was developed and used at the same location as the Aquadopp during the second deployment period. This alternative sensor, in the following referred with the name "pneumatic sensor", consists of a small steel box fixed on the sea floor with an open bottom. The variation of the pressure of the entrapped air inside the box, due the change of the water column above, is measured by a pressure transducer. From the pressure variation of the air inside the box, the water surface elevation can be obtained [7] as:

$$
\eta_{w}=\frac{p_{h y d}}{\rho g}
$$

where $p_{\text {hyd }}$ represents the dynamic water pressure at $60 \mathrm{~m}$ in front of the OWC, $\rho$ is the density of the sea water and $g$ is the gravitational acceleration. The data available from the Aquadopp sensor consist of 25 hours of records, while the data provided by the pneumatic sensor consist of 10 hours of records. The sampling frequency for both the deployment periods is equal to $2 \mathrm{~Hz}$.

The spectra of the chamber wave elevation measured during the first and second deployment period are reported in Figures $2 \mathrm{a}$ and $2 \mathrm{~b}$ respectively, while the corresponding spectra of the up-wave elevation measured with the Aquadopp and pneumatic sensor are reported respectively in Figures $2 \mathrm{c}$ and $2 \mathrm{~d}$. The spectra of the chamber wave elevation shows a resonance around $0.62 \mathrm{rad} / \mathrm{sec}$ for both deployment periods, which corresponds to a dominant wave period of approximately 10 seconds. However, the spectra of the free surface elevation up-wave of the WEC shows that, in addition to the dominant wave frequency, other components exist at higher frequency. Because the incident waves are reflected by the wall of the OWC, frequency components of shorter period can be present in the spectra of up-wave elevation [7].

Since the low frequency components carry most of the wave energy, and the dynamics of the chamber filters the high frequency components of the chamber wave elevation, it is a reasonable assumption to predict only the low frequency components of the chamber wave elevation [3]. Therefore the data are filtered with an ideal zero-phase low-pass filter with cut-off frequency $\omega_{c}$.

If it is assumed that the free surface elevation up-wave of the WEC propagates only in one direction, after a time delay called propagation time, the effect of the incident wave elevation is measured in the chamber. The propagation time can be inferred from consideration of wave propagation in shallow water. For shallow water with depth $h$, the waves can reach a maximum velocity $v_{\max }=\sqrt{g h}$, where $g$ is

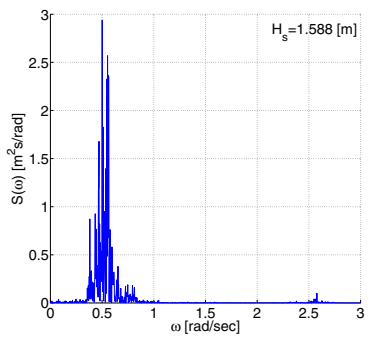

(a)

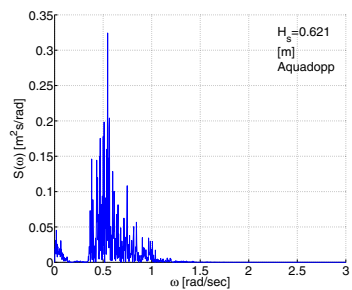

(c)

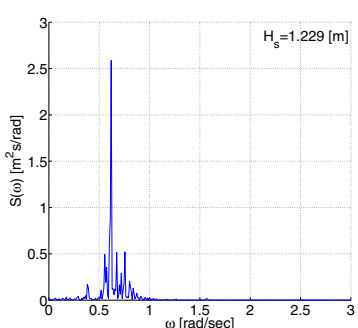

(b)

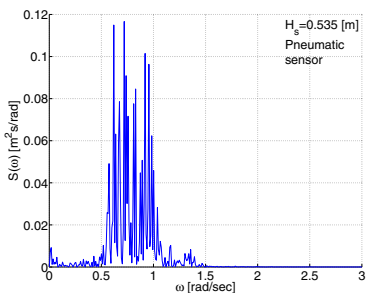

(d)
Fig. 2. Chamber wave elevation spectra for first (a) and second (b) deployment period and up-wave elevation spectra from Aquadopp sensor (c) and from pneumatic sensor (d)

the gravitational acceleration. Since the up-wave sensor is placed at a location with a water depth $h=8 \mathrm{~m}$, a velocity $v_{\max }=8.85 \mathrm{~m} / \mathrm{s}$ is obtained. If it is assumed that all waves travel at the same phase velocity equal to the maximum velocity, then the free surface elevation up-wave of the WEC reaches the chamber without being distorted. In shallow water, $k h \ll 1$, where $k$ is the wave number. For the range of frequencies of interest for our study, $k h \cong 0.5$. If $d$ denotes the spatial distance between the point of measurement of the free surface elevation up-wave of the WEC and the point of measurement of the chamber elevation, the propagation time $t^{*}$ of the wave is as follows [11]:

$$
t^{*}=\frac{d}{v_{\max }}
$$

Since the point of measurement of the up-wave elevation is placed at a distance $\mathrm{d}=60 \mathrm{~m}$ from the chamber, using equation (2), a propagation time around 7 seconds is obtained. In order to verify the propagation time computed using equation (2), a cross-correlation analysis between up-wave measurement and chamber wave elevation was performed, and a time-lag around 9 seconds was obtained.

\section{Forecasting Models}

\section{A. Up-Wave Only Models}

In this section, a model for forecasting the chamber wave elevation based only on the measurement of the free surface elevation up-wave of the WEC is presented. In particular, a Finite Impulse Response (FIR) model is designed which assumes that the chamber wave elevation $\eta_{c h}$ at time instant $k$ is a linear combination of $n_{b}$ past values of the free surface elevation up-wave of the WEC. The up-wave measurement is considered to be the input of the model and is denoted as $u$. The model considered is of the following form: 


$$
\eta_{c h}(k)=\sum_{i=1}^{n_{b}} b_{i} u\left(k-i+1-n_{k}\right)+\xi(k)
$$

where $\xi$ is considered to be a white noise with zero mean and variance $\sigma^{2}$. The term $n_{k}$ is the delay (in number of sample periods) that occurs before the output is affected by the input. Based on the propagation time and cross-correlation analysis performed in section 2 , the data coming from the upwave sensor are delayed opportunely, and therefore at time instant $k$, the water level in the chamber is influenced by the up-wave elevation at the same time instant. Therefore, the delay $n_{k}$ is considered to be zero. The FIR filter models the dynamics of the sensor utilized for the measurement of the free surface elevation up-wave of the WEC. In fact, since the up-wave sensor deployed approximates the free surface elevation based on a pressure signal, the FIR filter also models the dynamic relationship between the pressure and the free surface elevation. Given a set of parameters $b_{i}$, from equation (3) the $l$-step ahead prediction is given as follows:

$$
\hat{\eta}_{c h}(k+l \mid k)=\sum_{i=1}^{n_{b}} b_{i} u(k+l-i+1)
$$

Under the assumption of shallow water, the first term in equation (4) represents the value of the future chamber wave elevation for a forecasting horizon less or equal to the propagation time.

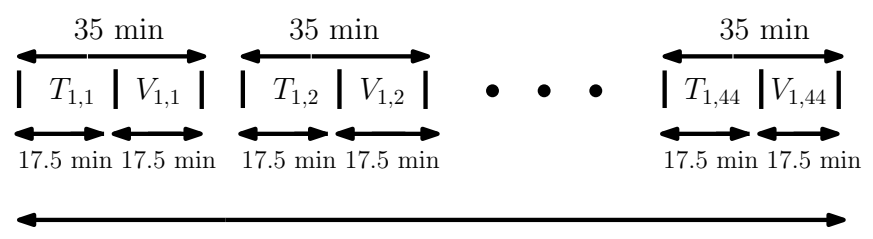

Period 1

(a)

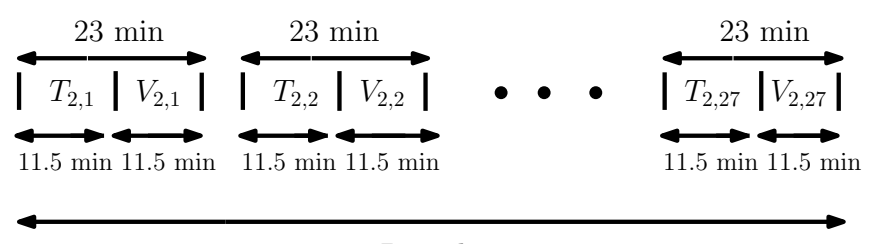

Period 2

(b)

Fig. 3. Time series data segmentation for the first (a) and second (b) deployment period

As shown in Figures $3 a$ and $3 b$, the data for each deployment period was divided into training and validation sets. A different model was identified and validated on each segment of 35 minutes of data for the first deployment period since the wave characteristics change value in different data segments. For the second deployment period, the data are representative of a more constant sea state, and the wave characteristics do not vary significantly during the registration time. The coefficients $b_{i}$ of the FIR model, at an instant $k$, are estimated using a batch of training data $T_{i, j}$ of dimension $N_{1}$ consisting of past observations of the water level in the chamber and measurements of the free surface elevation up-wave of the WEC. For the estimation of the model parameters, the cost function to be minimized is the variance of the one-step ahead prediction error, which is given as follows:

$$
J_{L S}=\sum_{k=1}^{N_{1}-1}\left(\eta_{c h}(k+1)-\hat{\eta}_{c h}(k+1 \mid k)\right)^{2}
$$

which is a linear Least Squares (LS) problem. For the estimation of the model parameters, a different cost function referred as Long Range Predictive Identification (LPRI) [12] was considered, with initial value of the parameters given by the minimization of (5). Since the minimization of the LPRI cost function for different forecasting horizons did not yield a significantly different value of the parameters compared to their initial estimates, the LPRI cost function was not further considered. An important element of the identification of the FIR model with cost function (5) is the choice of the order $n_{b}$. The objective is to identify a model that guarantees an accurate fitting of the training data without incurring so called "over-fitting", or rather the identification of a overparametrized model which also interpolates the stochastic noise presents in the measurements. Given a previously unseen batch of validation data $V_{i, j}$ of dimension $N_{2}$ consisting of observations of the chamber and up-wave elevations, the accuracy of the identified model can be validated by means of the variance of the prediction error $\sigma_{l}^{2}$ for a forecasting horizon $l$ computed as follows:

$\sigma_{l}^{2}=\frac{1}{N_{2}} \sum_{k=1}^{N_{2}} \hat{e}(k+l \mid k)^{2}=\frac{1}{N_{2}} \sum_{k=1}^{N_{2}}\left(\eta_{c h}(k+l)-\hat{\eta}_{c h}(k+l \mid k)\right)^{2}$

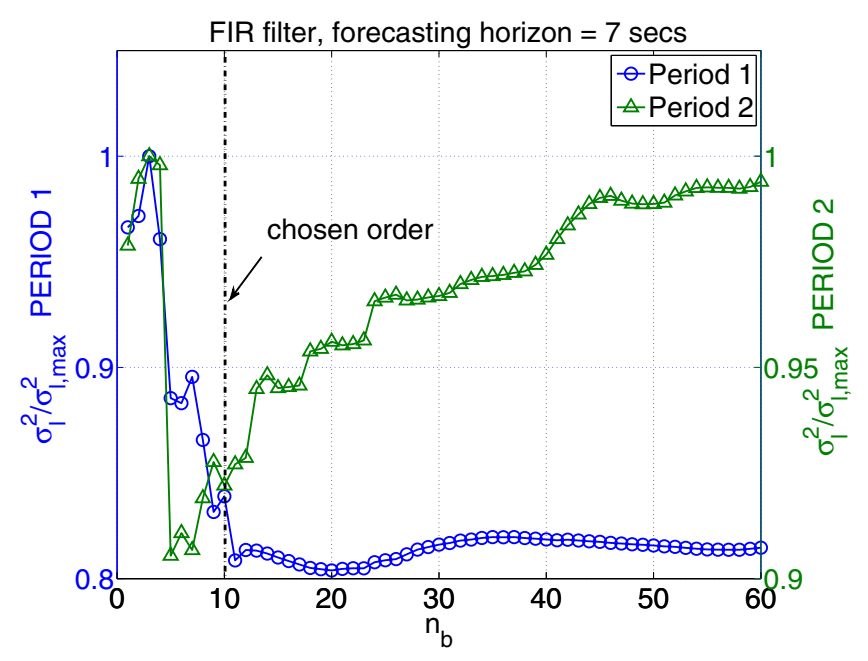

Fig. 4. Normalized prediction error variance for a forecasting horizon of 7 seconds as a function of the order $n_{b}$ averaged across validation data sets for the first and second deployment periods

In order to select the best order $\bar{n}_{b}$ that avoids overfitting the data, FIR filters of different orders $n_{b}$ were trained and validated by means of the data coming from 
the two deployment periods. In Figure 4, the variance $\sigma_{l}^{2}$ for a forecasting horizon equal to the propagation time of 7 seconds is computed for FIR filters of different order $n_{b}$. The variance is averaged across the validation data sets coming from both the deployment periods, normalized by the appropriate maximum value. An order $n_{b}$ equal to 10 is chosen as point of trade-off between the minima of the two curves.

\section{B. Autoregressive Models}

In this section, a model for forecasting the chamber wave elevation, based on its past history, is presented. In particular, an AutoRegressive (AR) model is proposed which assumes that the chamber wave elevation at time instant $k$ is linearly dependent on a number $n_{a}$ of its past values. Thus the model considered is of the following form:

$$
\eta_{c h}(k)=\sum_{i=1}^{n_{a}} \alpha_{i} \eta_{c h}(k-i)+\xi(k)
$$

Given a set of parameters $\alpha_{i}$, from equation (7) the $l$-step ahead prediction is given as follows:

$$
\hat{\eta}_{c h}(k+l \mid k)=\sum_{i=1}^{n_{a}} \alpha_{i} \hat{\eta}_{c h}(k+l-i \mid k)
$$

where $\hat{\eta}_{c h}(k+l-i \mid k) \equiv \eta_{c h}(k+l-i)$ if $k+l-i \leq k$, since the information is already available and there is no need to have a prediction [3]. The coefficients $\alpha_{i}$ of the AR model are estimated using the cost function given by equation (5), and the identified models can be validated according to the variance of the prediction error over a forecasting horizon $l$, given by equation (6). AR models of different orders $n_{a}$ were trained and validated by means of the measurements of the wave elevation inside the chamber for both the deployment periods. In Figure 5, the variance $\sigma_{l}^{2}$ for a forecasting horizon equal to 30 seconds, normalized by its maximum value and averaged across the validation sets, is plotted against different order $n_{a}$ for both the deployment periods. An order $n_{a}$ equal to 30 is chosen since it represents a minimum point for both the two curves.

\section{Combination Up-Wave/Autoregressive Models}

In this section, a model for forecasting the chamber wave elevation based on a combination of its past history and measurements of the free surface elevation up-wave of the WEC is presented. In particular, an AutoRegressive eXogenous input (ARX) model is proposed which assumes that the chamber wave elevation at time instant $k$ is linearly dependent on a number $n_{a}$ of its past values and on $n_{b}$ values of the up-wave measure. Thus, the model considered is of the following form:

$$
\eta_{c h}(k)=\sum_{i=1}^{n_{a}} a_{i} \eta_{c h}(k-i)+\sum_{i=1}^{n_{b}} b_{i} u(k-i+1)+\xi(k)
$$

Given a set of parameters $a_{i}$ and $b_{i}$, from equation (9), the $l$-step ahead prediction is given as follows:

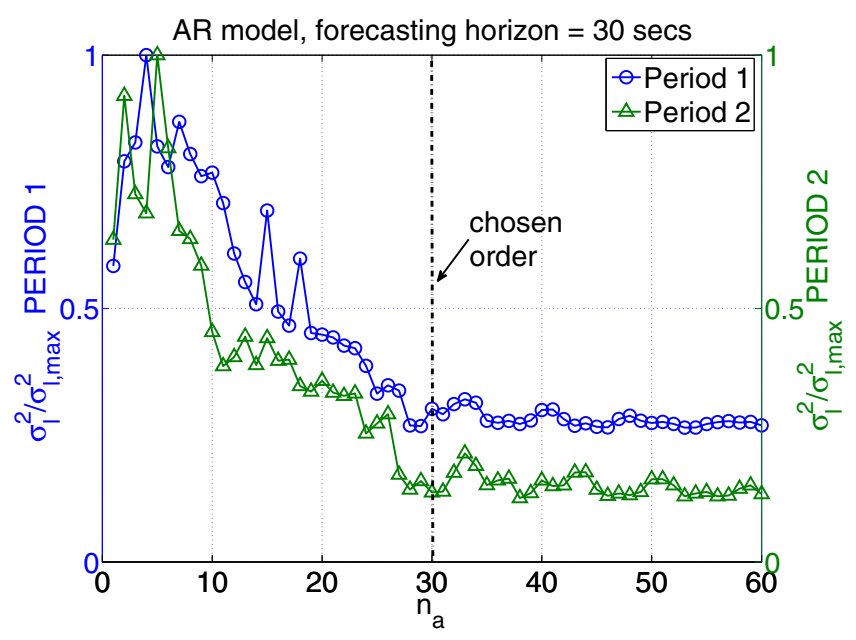

Fig. 5. Normalized prediction error variance for a forecasting horizon of 30 seconds as a function of the order $n_{a}$ averaged across validation data sets for the first and second deployment periods

$\hat{\eta}_{c h}(k+l \mid k)=\sum_{i=1}^{n_{a}} a_{i} \hat{\eta}_{c h}(k+l-i \mid k)+\sum_{i=1}^{n_{b}} b_{i} u(k+l-i+1)$

In equation (10), no prediction of the input is made until a prediction horizon, equal to the propagation time of the wave from the location of the sensor is reached. The limit of the prediction horizon in time steps will be denoted as $l_{\text {max }}$. Therefore, the prediction of $\hat{\eta}_{c h}$ can be made with an ARX model only until $l_{\text {max }}$ steps, after which an AR only model is used to predict the water level in the chamber. Thus, the complete prediction of the wave elevation using an ARX model is given as follows:

$$
\hat{\eta}_{c h}(k+l \mid k)=\left\{\begin{array}{cl}
\sum_{i=1}^{n_{a}} a_{i} \hat{\eta}_{c h}(k+l-i \mid k)+. . & \\
\sum_{i=1}^{n_{b}} b_{i} u(k+l-i+1) & \text { if } l \leq l_{\text {max }} \\
\sum_{i=1}^{n_{a}} \alpha_{i} \hat{\eta}_{c h}(k+l-i \mid k) & \text { else }
\end{array}\right.
$$

The coefficients $a_{i}, b_{i}$ of the ARX model and $\alpha_{i}$ of the AR model are estimated individually using the cost function given by equation (5), and the identified models were validated according to the variance of the prediction error, over a forecasting horizon $l$, given by equation (6).

Given an order $n_{a}=30$ for the AR model obtained in the previous section, ARX models of different orders $n_{b}$ were trained and validated for both the deployment periods. In Figure 6, the normalized variance of the prediction error, averaged across the validation data sets, is plotted against different orders $n_{b}$ for both the deployment periods. By inspection of Figure 6, an order $n_{b}=15$ is selected as a trade-off point between the minima of the two curves. The 
order $n_{b}$, chosen for the ARX model, is different from the order $n_{b}$ of the FIR model, since the ARX models were trained and validated for a fixed choice of $n_{a}=30$.

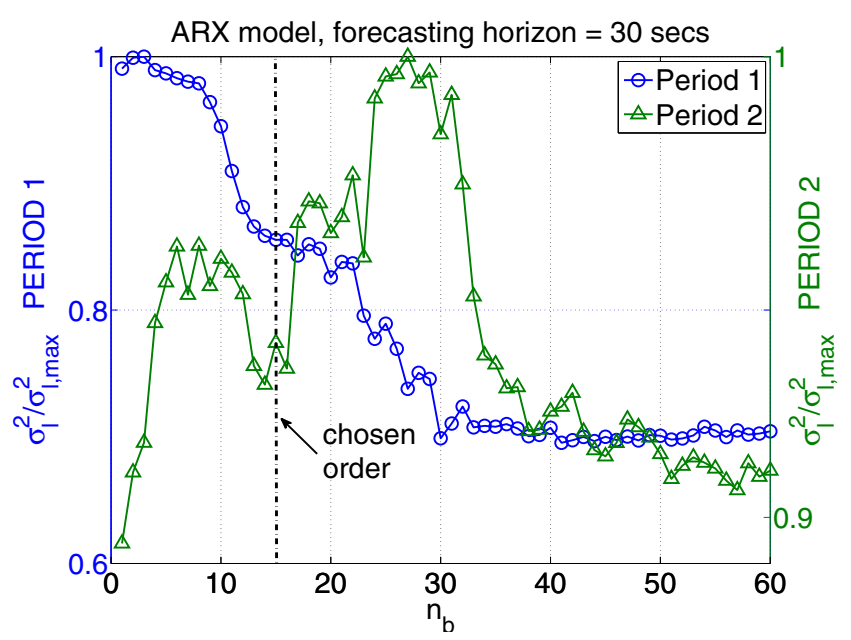

Fig. 6. Normalized prediction error variance for a forecasting horizon of 30 seconds as a function of the order $n_{b}$ with $n_{a}=30$ averaged across validation data sets for the first and second deployment periods

\section{RESUlts}

In the following section, the forecasting of the water level in the chamber given by the FIR and ARX models is compared with the forecasting given by the AR model over a certain range of prediction horizons. The index used for the prediction accuracy for every forecasting horizon $l$ is the Goodness Of Fit (GOF) index, which is defined as follows:

$$
G O F(l)=\left(1-\frac{\sqrt{\sum_{k}(\eta(k+l)-\hat{\eta}(k+l \mid k))^{2}}}{\sqrt{\sum_{k} \eta(k)^{2}}}\right) \cdot 100
$$

In order to compare the performance between the AR model and the FIR or ARX model, the Relative Goodness of Fit (RGOF) is introduced:

$$
R G O F_{F I R / A R X}(l)=G O F_{F I R / A R X}(l)-G O F_{A R}(l)
$$

The RGOF between an AR model of order $n_{a}=30$ and a FIR model of order $n_{b}=10$, averaged across the validation data sets, is plotted against the forecasting horizon for the first and second deployment period in Figure 7. As shown by Figure 7, the AR model is able to provide more accurate predictions of the wave elevation in the chamber for all forecasting horizons up to 7 seconds. In Figure 8, the RGOF between an AR model of order $n_{a}=30$ and a ARX model of order $n_{a}=30$ and $n_{b}=15$, averaged across the validation data sets of the first deployment period, is plotted against the prediction horizon for different values of the cut-off frequency $\omega_{c}$. As the figure shows, the AR model performs better than the ARX model for prediction horizons that range from 10 seconds up to 22 or 27 seconds, depending on $\omega_{c}$. The choice of the cut-off frequency $\omega_{c}$ depends on

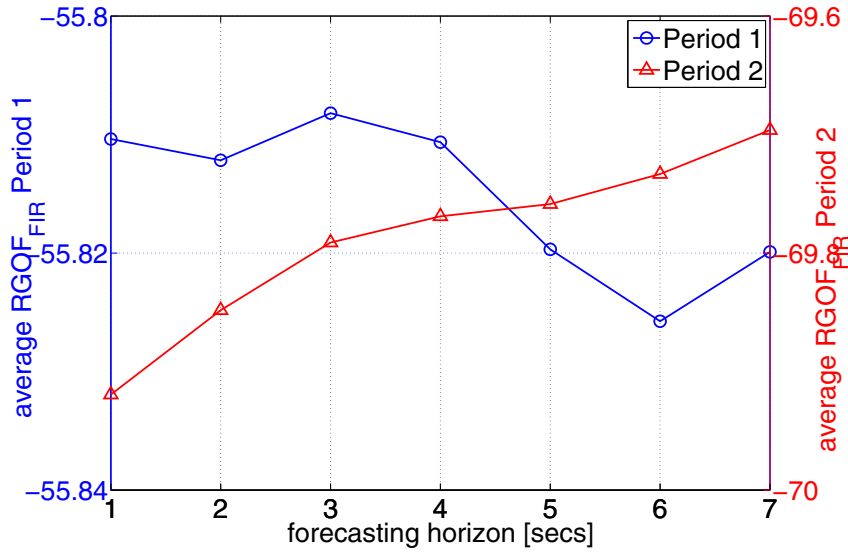

Fig. 7. Relative goodness of fitting (RGOF) between AR model $\left(n_{a}=30\right)$ and FIR model $\left(n_{b}=10\right)$ averaged across the validation data sets of the first and second deployment periods

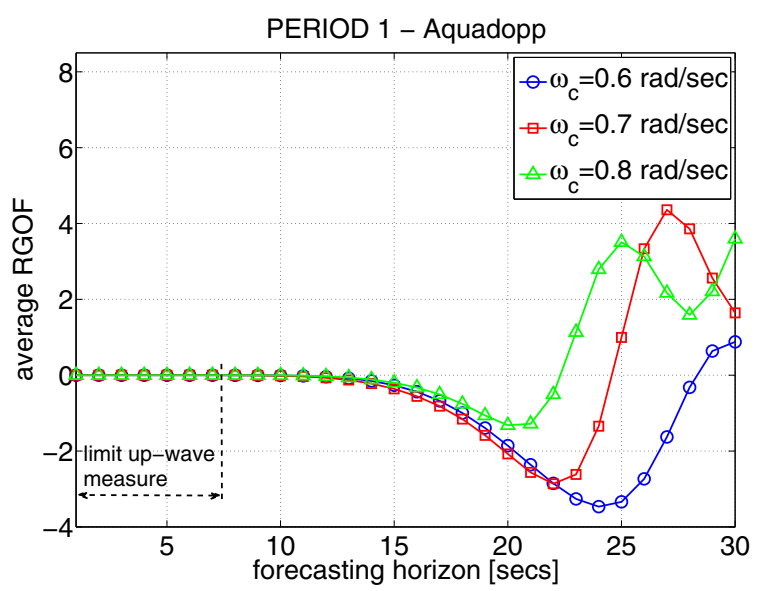

Fig. 8. Relative goodness of fitting (RGOF) between AR model $\left(n_{a}=30\right)$ and ARX model $\left(n_{a}=30, n_{b}=15\right)$ averaged across the validation data sets of the first deployment period

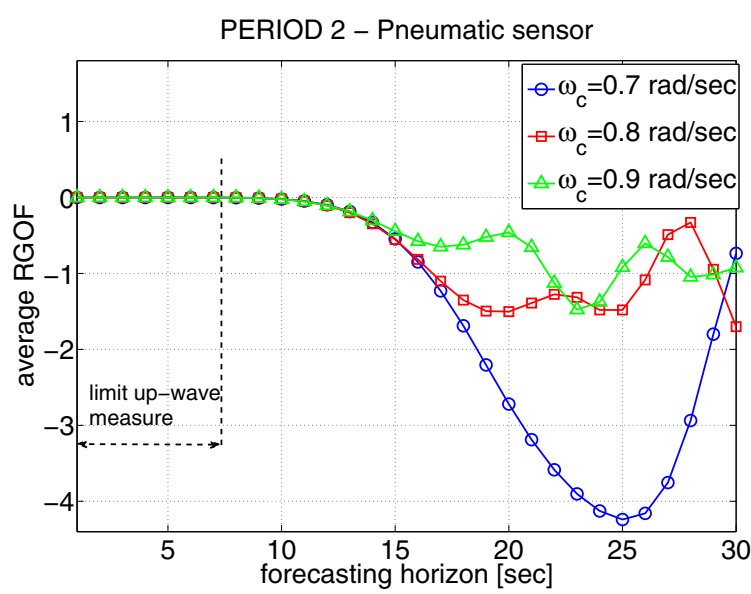

Fig. 9. Relative goodness of fitting (RGOF) between AR model $\left(n_{a}=30\right)$ and ARX model $\left(n_{a}=30, n_{b}=15\right)$ averaged across the validation data sets of the second deployment period

frequency components of the chamber wave elevation that need to be predicted. Since the resonance of the chamber 


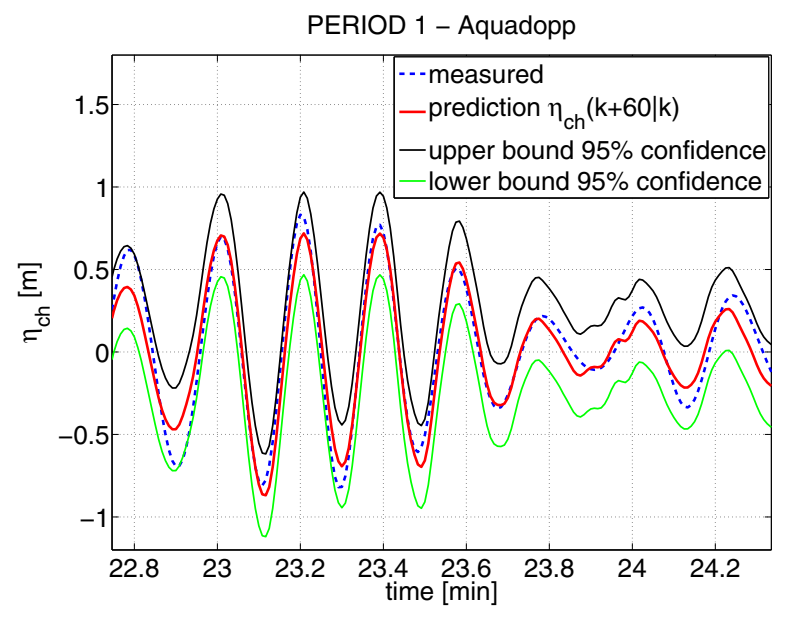

Fig. 10. Comparison between measured chamber wave elevation and prediction $\eta_{c h}(k+60 \mid k)$ computed with ARX model of order $n_{a}=30$ and $n_{b}=15$ with data set of first deployment period, filtered with a cut-off frequency of $\omega_{c}=0.7 \mathrm{rad} / \mathrm{sec}$

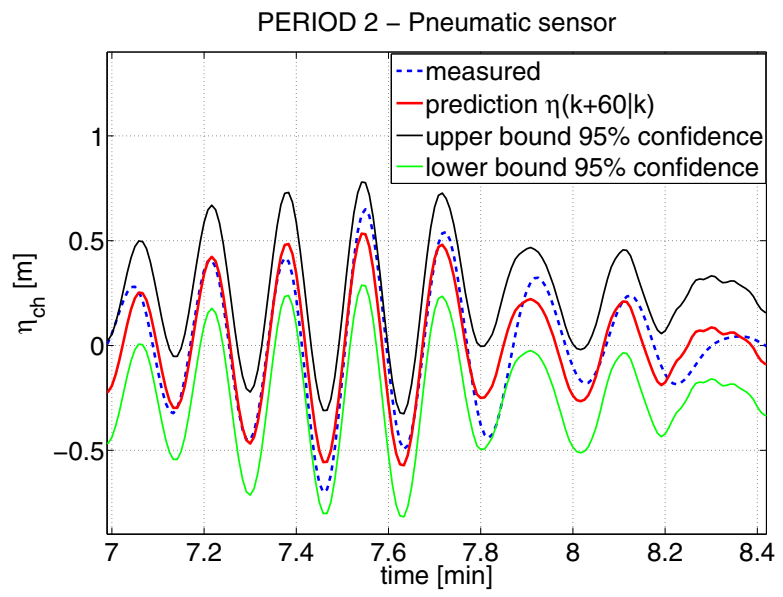

Fig. 11. Comparison between measured chamber wave elevation and prediction $\eta_{c h}(k+60 \mid k)$ computed with ARX model of order $n_{a}=30$ and $n_{b}=15$ with data set of second deployment period, filtered with a cut-off frequency of $\omega_{c}=0.7 \mathrm{rad} / \mathrm{sec}$

is around $0.6 \mathrm{rad} / \mathrm{sec}$, a cut-off frequency $w_{c}=0.7 \mathrm{rad} / \mathrm{sec}$ is selected for filtering the chamber and up-wave elevation. Regarding the second deployment period, in Figure 9, the RGOF between an AR model of order $n_{a}=30$ and an ARX model of order $n_{a}=30$ and $n_{b}=15$, averaged across the validation data sets, is plotted against the prediction horizon for different values of the cut-off frequency $\omega_{c}$. As the figure shows, the ARX model provides less accurate predictions of the wave elevation inside the chamber than the AR model for every prediction horizon. For forecasting horizons longer than 7 seconds, the RGOF is different from zero even though the forecasting model in equation (8) and (11) employ an AR model for the prediction of the wave elevation. Generally, the AR and ARX model compute different predictions of the chamber wave elevation for forecasting horizons shorter than 7 seconds, and as a result, the two forecasting models have different initial conditions for forecasting horizons longer than 7 seconds. A comparison between the measured chamber wave elevation and the prediction of the chamber wave elevation for a forecasting horizon of 30 seconds given by an ARX model of order $n_{a}=30$ and $n_{b}=15$ is made. The comparison utilizes the free surface elevation up-wave of the WEC provided by the Aquadopp and pneumatic sensors, reported in Figures 10 and 11 respectively.

\section{CONCLUSIONS}

This paper is focused on the prediction of the wave elevation inside the chamber of the OWC located on Pico Island. Initially the prediction of the chamber wave elevation was obtained by means of an approach based purely on the free surface elevation up-wave of the WEC. In particular, a FIR filter was deployed and its performance was compared with the performance of an AR model relying only on the past values of the chamber wave elevation. The comparison showed that the spatial approach gives less accurate predictions respect to the AR model. Additionally, an ARX model was tested which predicts the future wave elevation in the chamber based on the combination of its past values and measurements of the up-wave elevation. This comparison showed that the ARX model does not significantly improve the accuracy of the wave prediction given by the AR model.

\section{ACKNOWLEDGMENT}

This paper is based upon works supported by the Science Foundation Ireland under Grant No. 12/RC/2302 for the Marine Renewable Ireland (MaREI) centre.

\section{REFERENCES}

[1] U. A. Korde, "Control system applications in wave energy conversion," in Proc. OCEANS 2000 MTS/IEEE Conf. Exhibition, vol. 3, USA, 2000, pp. 1817-1824.

[2] J. Falnes, Ocean Waves and Oscillating Systems. Cambridge, U.K.: Cambridge University Press, 2002.

[3] F. Fusco and J. V. Ringwood, "Short-term wave forecasting for real-time control of wave energy converters," IEEE Transactions on Sustainable Energy, vol. 1, no. 2, pp. 99-106, July 2010.

[4] J. Tedd and P. Frigaard, "Short term wave forecasting, using digital filters, for improved control of wave energy converters," in Proc. International Offshore and Polar Engineering Conference, 2007, pp. 388-394.

[5] J. Dannenberg, K. Hessner, P. Naaijen, H. V. D. Boom, and K. Reichert, "The on board wave and motion estimator owme," in Proc. International Offshore and Polar Engineering Conference, 2010, pp. 424-431.

[6] M. R. Belmont, J. M. K. Horwood, R. W. F. Thurley, and J. Baker, "Shallow angle wave profiling lidar," Atmospheric and Oceanic Technology, vol. 24, no. 6, pp. 1150-1156, 2007.

[7] K. Monk, D. Conley, M. Lopes, and Q. Zou, "Pneumatic power regulation by wave forecasting and real-time relief valve control for an owc," in Proc. of the European Wave and Tidal Energy Conference Series, Aalborg, Denmark, 2013.

[8] B. Fischer, P. Kracht, and S. Perez-Becker, "Online-algorithm using adaptive filters for short-term wave prediction and its implementation," in Proc. International Conference on Ocean Energy, 2012.

[9] M. P. Schoen, K. Hals, and T. Moanr, "Wave prediction and robust control of heaving wave energy devices for irregular waves," IEEE Transactions on Energy Conversion, vol. 26, no. 2, pp. 627-638, 2011.

[10] A. Price and A. Wallace, "Non-linear methods for next wave estimation," in Proc. 7th European Wave and Tidal Energy Conference, 2007.

[11] U. A. Korde, "Up-wave surface elevation for smooth hydrodynamic control of wave energy conversion in irregular waves," in Proc. of the OCEANS'13 San Diego, 2013.

[12] D. S. Shook, C. Mohtadi, and S. Shah, "Identification for long-range predictive control," in Proc. Inst. Elect. Eng., D Control Theory and Applications, vol. 138, no. 1, 1991, pp. 75-84. 